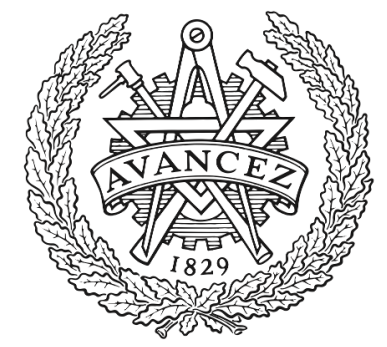

\title{
CHALMERS
}

UNIVERSITY OF TECHNOLOGY

\section{Simple Formula for Aperture Efficiency Reduction Due to Grating Lobes in Planar Phased Arrays}

Downloaded from: https://research.chalmers.se, 2023-04-26 12:54 UTC

Citation for the original published paper (version of record):

Vosoogh, A., Kildal, P. (2016). Simple Formula for Aperture Efficiency Reduction Due to Grating Lobes in Planar Phased Arrays. IEEE Transactions on Antennas and Propagation, 64(6): 2263-2269. http://dx.doi.org/10.1109/TAP.2016.2551259

N.B. When citing this work, cite the original published paper.

C2016 IEEE. Personal use of this material is permitted.

However, permission to reprint/republish this material for advertising or promotional purposes 


\title{
Simple Formula for Aperture Efficiency Reduction Due to Grating Lobes in Planar Phased Arrays
}

\author{
Abbas Vosoogh, Per-Simon Kildal, Fellow, IEEE
}

\begin{abstract}
This paper numerically validates a simple formula to calculate the aperture efficiency reduction due to grating lobes in planar phased arrays. Array antennas with element spacing greater than one wavelength will produce grating lobes. Grating lobes are not a big problem in most new millimeter wave applications, except for the fact that they reduce the aperture efficiency and thereby normally the directivity. When the element pattern is known, the simple formula can be used to calculate the aperture efficiency in the presence of grating lobes. The formula is verified by simulations of a large uniformlyexcited planar slot array. The agreement is good both for broadside radiation and a phased main beam, even if we use the far-field of an isolated slot. Using the far-field of the embedded slot element gives almost the same results, except when a grating lobe radiates along the ground plane and the isolated element pattern is non-zero in this direction.
\end{abstract}

Index Terms - aperture efficiency, array antenna, grating lobe

\section{INTRODUCTION}

$\mathrm{P}$ lanar array antennas are very popular and widely used because of their advantages such as flat structure, low volume and weight. Furthermore in array antennas the main beam direction can be rapidly changed by electronic steering. This makes them attractive for many applications. Usually in the design of array antennas, the element spacing is required to be within one wavelength in order to avoid high grating lobes. However, the element spacing may become larger than one wavelength, especially at high frequencies. Several methods have been developed to suppress grating lobes, such as aperiodic arrays configurations including rotated sub arrays [1], ring-grid array with trapezoid subarrays [2], arrays of random subarrays [3], random element and subarray positioning [4], and processing techniques in synthetic aperture radar (SAR) systems [5]-[6].

The continuously growing demands for higher data rate communication lead to using higher frequency bands, e.g. in applications such as automotive, radar and communication

A. Vosoogh and P.-S. Kildal are with the Signals and Systems Department at Chalmers University of Technology, 41296 Gothenburg, Sweden, e-mail: abbas.vosoogh@chalmers.se,per-simon.kildal@chalmers.se.

The work has been financially supported by the European Research Council (ERC) under the $7^{\text {th }}$ Framework Program ERC grant number 321125 and Swedish Governmental Agency for Innovation Systems (VINNOVA) within the VINN Excellence Center Chase at Chalmers. system terminals. The gap waveguide technology presents some benefits for high frequency antenna applications [7]. It has a planar profile, and it can be used as low loss distribution network for an antenna array. The width of the lines can be increased to reduce the conductive losses and there is no problem with excitation of surface waves, which severely affect common microstrip distribution networks at high frequency [8]. However, the element spacing may become larger than one wavelength in order to accommodate a corporate distribution network [9], and this may cause grating lobes. For millimeter waves, the attenuation in the atmosphere is large, so grating lobes are not a serious problem in terms of interference. Still, they cause reduction of the directivity and aperture efficiency, and this is undesired in itself. Therefore, the purpose of this study is to determine the grating lobe levels and their effect on the aperture efficiency.

Large array antennas can be designed as an array of subarrays [10]. Grating lobes will also appear when using subarrays, caused by all types of variations within the subarray (i.e. unit cell) that causes variations along the array of period equal to the period of the subarray, such as: uneven distribution of the elements within each subarray, and amplitude and phase variations between the excitations of the elements within each subarray. In [11] a $2 \times 2$ - cavity-backed slot subarray is used as a directive array element, designed for suppressing grating lobes due to the large 2 lambda element spacing applied to reduce losses in the distribution network. Because of odd excitation of the slots due to the higher order mode in the cavity, the grating lobes appeared in the radiation pattern. Reference [12] categorized and demonstrated specific subarray pattern distortions that can cause grating lobes appearance in uniform arrays. Reference [13] shows how dielectric superlayers can be used to reduce the grating lobe level in thinned phase arrays.

In the present paper we make a generic study of grating lobes in a large slot array. A simple formula is presented for aperture efficiency reduction due to the grating lobes, see Chapter 10 in [14]. We numerically verify this simple formula for a uniformly excited $32 \times 32$-element array of slots in an infinite ground plane. We show that even though the embedded element far-field function is needed in order to calculate the grating efficiency, the isolated far-field pattern is sufficient except when a grating lobe radiates along the ground plane and the isolated element pattern is non-zero in this 
direction. However, for this case there is normally no need to know the accurate gain, only a need to detect the presence of blindness, so in most cases the isolated element approach also works well enough then.

\section{GRATING EFFICIENCY}

Traditionally, the antenna aperture efficiency is defined as the ratio between the directivity $\mathrm{D}$ of the antenna and the maximum available directivity $\mathrm{D}_{\max }$, i.e.

$$
e_{a p}=D / D_{\max } .
$$

The maximum achievable directivity from a large planar antenna aperture is given by:

$$
D_{\max }=\frac{4 \pi A}{\lambda^{2}}
$$

where $\mathrm{A}$ is the area of the aperture evaluated as the number of elements times the area of the unit cell in the planar arrays. In order to calculate the aperture efficiency with this formula, we just need to know the directivity of the antenna.

In an array antenna, when the element spacing becomes greater than one wavelength, the first grating lobes are in the visible region for a broadside scan. These grating lobes can lead to antenna directivity reduction. The level of the grating lobes and consequently the directivity reduction depend on the element far-field function and the element or subarray spacing. Therefore, in order to calculate the aperture efficiency in the presence of grating lobes by using (1), the directivity must first be determined. We will here do this by a numerical approach.

A simple formula called "grating efficiency" is presented in [14, Sec.10.3.5]. The grating efficiency is the reduction in directivity and consequently it is the aperture efficiency due to the power lost in the grating lobes. It is given by the following formula:

$$
e_{g r t}=\frac{\left|\mathbf{G}\left(\theta_{0}, \varphi_{0}\right)\right|^{2}}{\sum_{p q}\left|\mathbf{G}\left(\theta_{p q}, \varphi_{p q}\right)\right|^{2} \frac{\cos \theta_{0}}{\cos \theta_{p q}}}
$$

The sum is taken over all visible grating and main lobes. In (3) $\mathbf{G}(\theta, \varphi)$ is the vector far-field function of the single embedded element, $\left(\theta_{0}, \varphi_{0}\right)$ is the direction of main beam and $\left(\theta_{p q}, \varphi_{p q}\right)$ are the directions of the grating lobes. The embedded element far-field function is the far-field function of the whole array when only one element is exited and all the other elements are terminated in the port impedance. This formula is derived under the assumptions of, firstly, all grating lobes being in the visible region and no grating lobe being at grazing angle, secondly, the array being large and having a pencil beam, and finally, the edge effects are approximated by assuming that all elements have the same embedded radiation pattern. This is in practice possible by adding a few dummy rows of elements around the excited part of the array. The directions of the grating lobes can be calculated by [14,
Sec.10.3.4]:

$$
\left\{\begin{array}{l}
\sin \theta_{p q} \cos \varphi_{p q}=\sin \theta_{0} \cos \varphi_{0}+p \frac{\lambda}{d_{x}} \\
\sin \theta_{p q} \sin \varphi_{p q}=\sin \theta_{0} \sin \varphi_{0}+q \frac{\lambda}{d_{y}}
\end{array}\right.
$$

These are grating lobes for $\mathrm{p}= \pm 1, \pm 2, \ldots$ and $\mathrm{q}= \pm 1, \pm 2, \ldots$, where $d_{x}$ and $d_{y}$ are the element spacing in the $x$ and $y$ direction, respectively, and $\left({ }_{0}, 0\right)$ is the direction of the main beam. With the simple formula (3) and the knowledge of the radiation pattern of the element, we can analytically estimate the aperture efficiency and therefore the directivity of the array, in the presence of grating lobes. We will also evaluate the accuracy of the grating efficiency formula by comparison with numerical and analytical solutions.

\section{ELEMENT FAR-FIELD FUNCTION}

A single half-wavelength slot in an infinite ground plane is used as the array unit cell. It has a well-known far-field function, and therefore we can easily calculate the grating efficiency of the array antenna analytically. Thereby it represents a clean and pedagogical example. Slots are also often used in practical planar arrays. Fig. 1 shows the slot unit cell geometry. The slot has a length of $0.505 \lambda$ and a width of $0.067 \lambda(<<\lambda)$.It is placed in the $x y$ plane with the element spacings $d_{x}$ and $d_{y}$ in the $\mathrm{x}$ and $y$ directions, respectively.

We can find the far-field function of an isolated slot in an infinite ground plane by using an equivalent magnetic current source [14, Sec. 5.4.1]:

$$
\mathbf{G}_{s l t}(\hat{\mathbf{r}})=2 E_{0} w \mathbf{G}_{i m g}(\hat{\mathbf{r}}) \tilde{M}(k \hat{\mathbf{x}} \cdot \hat{\mathbf{r}})
$$

the factor $w E_{0}$ is the voltage over the center of slot and $\mathbf{G}_{\mathrm{img}}$ is the vector far-field function of an incremental magnetic current source. For a $y$-polarized slot antenna, the latter becomes [14, Sec. 4.4.4]:

$$
\hat{\mathbf{I}}_{m}=-\hat{\mathbf{x}}: \rightarrow \mathbf{G}_{i m g}=C_{k}\lfloor\sin \varphi \hat{\theta}+\cos \theta \cos \varphi \hat{\varphi}\rfloor
$$

In (6) $C_{k}=-j k / 4 \pi$ where $k$ is the wave number and finally $\tilde{M}(k \hat{x} \cdot \hat{r})$ is the Fourier transform of the magnetic current distribution along the slot, i.e.

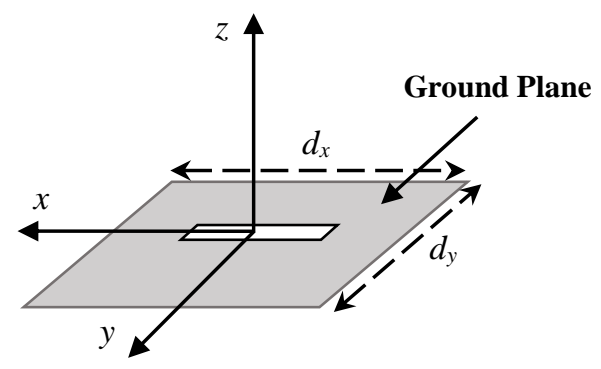

Fig. 1. Slot unit cell geometry. 


$$
\hat{M}(k \hat{\mathbf{r}} \cdot \hat{\mathbf{x}})=\frac{2}{k} \cos \left(\frac{\pi}{2} \sin \theta \cos \varphi\right) /\left[1-(\sin \theta \cos \varphi)^{2}\right]
$$

By substituting (5) into the grating efficiency formula, we can calculate the aperture efficiency of the slot array, analytically when all elements are excited by the same amplitude and a linearly varying phase in order to provide the desired main beam direction $\left(\begin{array}{ll}0 & 0\end{array}\right)$.

\section{NUMERICAL AND ANALYTICAL RESULTS}

We considered a planar $32 \times 32$-element slot array with uniform rectangular grid and uniform excitation. The appearances of grating lobes are determined by the element spacing in E-plane and H-plane ( $y$ and $x$ direction, respectively). When the element spacing is slightly smaller than one wavelength, the first grating lobe starts to appear in the visual region. In order to verify the accuracy of the presented formula, the numerical and analytical results of the grating efficiency have been compared with the aperture efficiency obtained from full-wave numerical simulations.

For our numerical approach, the single element has been simulated in the infinite array environment by applying the Master/Slave boundary condition in HFSS. The waveguide port excitation is used to feed the unit cell. By using this combined with a truncation to $32 \times 32$ elements (done by

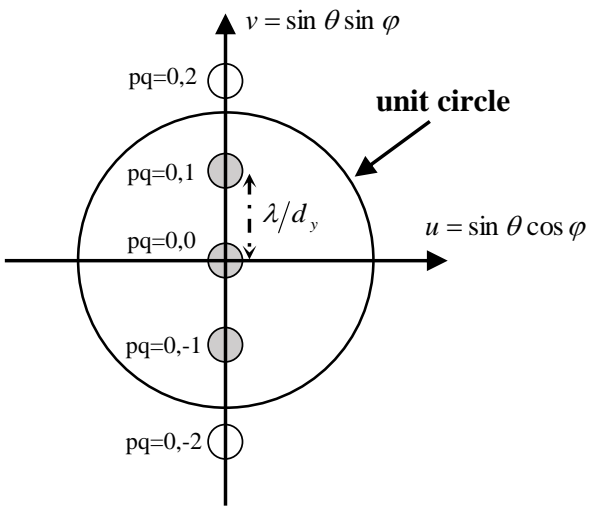

Fig. 2. Grating lobes and main beam spectrum for element spacing greater than one wavelength in E-plane.

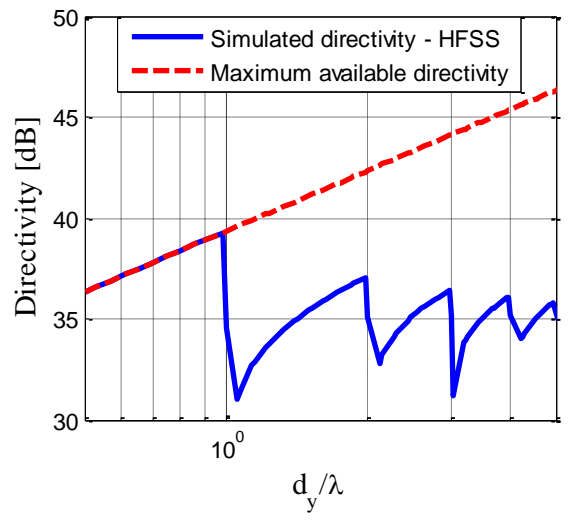

Fig. 3. Directivity of $32 \times 32$-elemnt slot array with different element spacing in E-plane.
HFSS), we obtained the directivity and the far-field function of the $32 \times 32$ array for different element spacings. Then the aperture efficiency is calculated by using (1). These results are in the graphs marked with "e $e_{a p}$ - Numerical". Furthermore the grating efficiency is calculated by substituting the level of the main beam and grating lobes obtained from the simulated radiation patterns into the grating efficiency formula. These results are marked with "e grt - Numerical".

One advantage of the grating efficiency formula in (3) is that we can calculate the aperture efficiency of a large array in the presence of grating lobes, analytically. For this aim we need the embedded element pattern. Computing the embedded element pattern is not simple and the unit cell needs to be simulated embedded in its environment. However, we will also show that knowing the isolated element pattern in (5) is a good approximation for most element spacings. These curves are marked "e $e_{\text {ap }}$ - Analytical".

We will now present directivity and efficiency results in the presence of grating lobes versus element spacing for different element spacings with the approaches described above.

\section{A. Grating Lobes in E-plane}

In this case we assumed that grating lobes only appear in Eplane. To satisfy our assumption, the element spacing in $\mathrm{H}$ plane is smaller than one wavelength. The element spacing in E-plane varies from $0.1 \lambda$ to $10 \lambda$ while the element spacing is

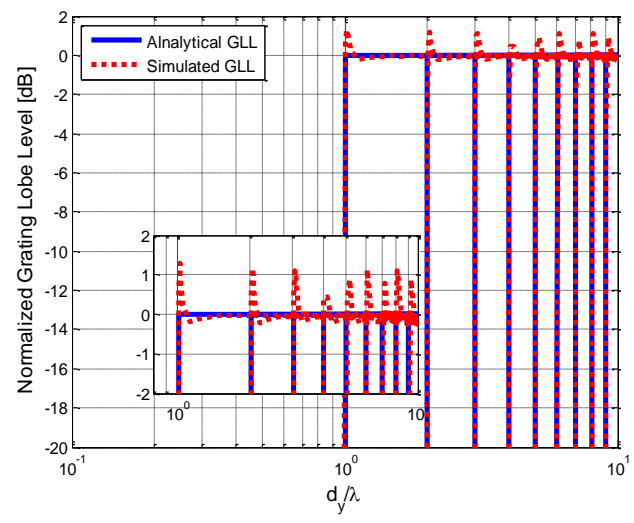

Fig. 4. Grating lobe level of $32 \times 32$-element slot array with different element spacing in E-plane.

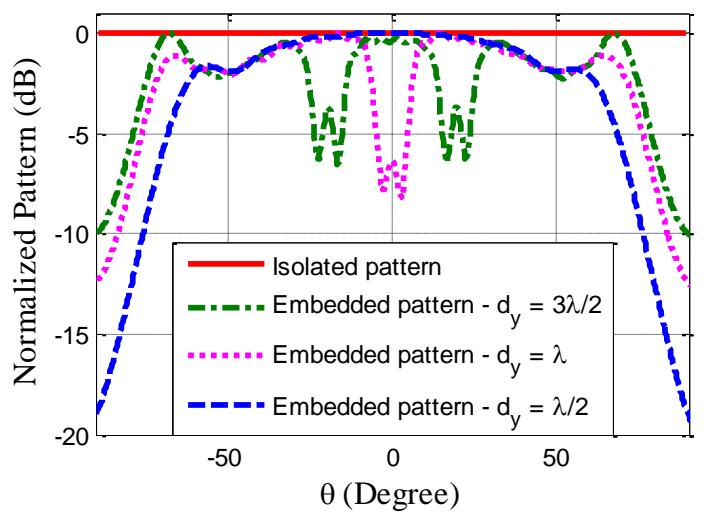

Fig. 5. Comparison of isolated and embedded element pattern with different element spacing in E-plane. 


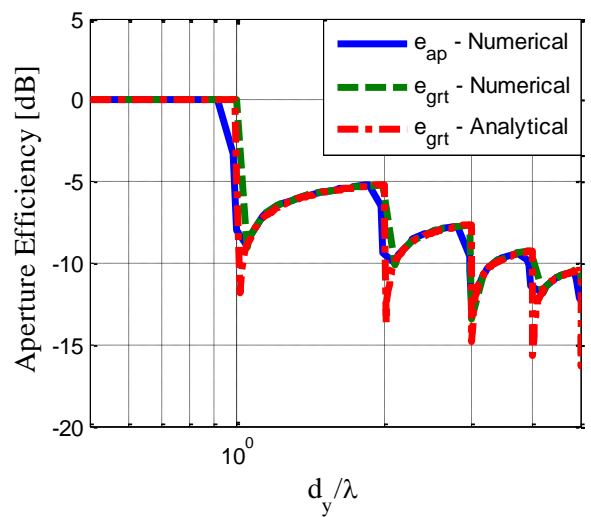

Fig. 6. Aperture efficiency of $32 \times 32$-element slot array with different element spacing in E-plane.

fixed to $d_{x}=0.67 \lambda$ in H-plane ( $x$-direction).

The spectrum of grating lobes for this case is shown in Fig. 2 , which shows the grating lobe location in $u v$-coordinates for an in-phase rectangular grid array. The locations of grating lobes are determined by the element spacing in E-plane $\left(d_{y}\right)$ and the grating lobes inside the unit circle radiate.

Fig. 3 shows the simulated directivity for the $32 \times 32$ slot array and the maximum available directivity of an array with the same size calculated using (2). When the element spacing is an integer number of wavelengths $\left(d_{y} / \lambda=1,2,3, \ldots\right)$, two new grating lobes (one on each side of the broadside main beam) start to appear in the visual region, and therefore the array directivity drops severely. This is because the beamwidth of the grating lobes is much wider than the beamwidth of the broadside main beam, when the grating lobe appears along the ground plane of the array. Furthermore, they are not suppressed by the element patterns, which is almost uniform in E-plane.

The normalized grating lobe levels obtained by HFSS are in Fig. 4 compared with the levels based on the analytical model for the far-field function of an isolated slot in (5). When the element spacing is an integer number of wavelengths, new grating lobes appear in the visual region. According to (5), (6) and (7) the isolated element pattern of the slot is almost uniform in E-plane. Therefore, the normalized analytical grating lobe level is equal to one for all grating lobes. The

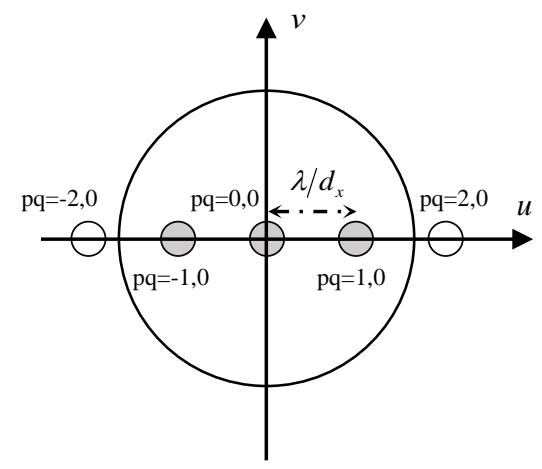

Fig. 7. Grating lobes and main beam spectrum with different element spacing in $\mathrm{H}$-plane

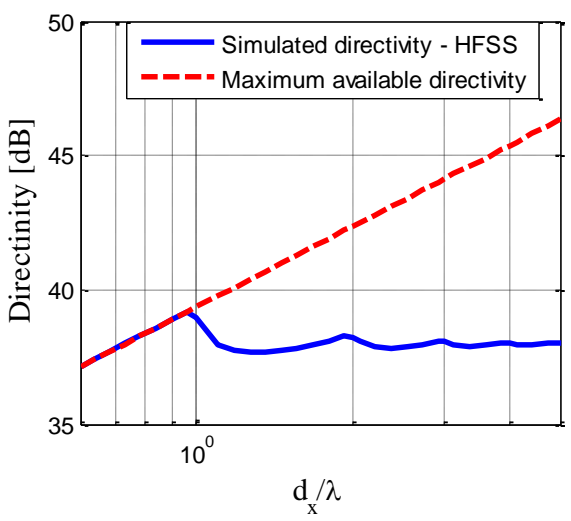

Fig. 8. Directivity of $32 \times 32$-element slot array with different element spacing in H-plane.

analytical and simulated grating lobe levels show good agreement with each other except when there are grating lobe directions near to the end-fire directions (along the ground plane). The reason for this difference is that we used the isolated element pattern instead of the embedded element pattern. According to this figure, the isolated element pattern is quite accurate for calculating the grating lobe level except when there are grating lobes radiating along the ground plane. Fig. 5 shows a comparison between the isolated and embedded element patterns in E-plane. Because of the high mutual coupling between slots in E-plane, the isolated and embedded patterns are very different. Furthermore, the embedded element pattern change when the element spacing changes. As mentioned before, computing the embedded element pattern is difficult, but as shown in Fig. 4, the isolated element pattern can be used to calculate the grating efficiency with good accuracy except for main beam directions at which grating lobes suddenly appear along the array. These are the directions corresponding to the dips in the embedded element patterns, i.e. directions associated with blindness. However, the grating efficiency predicts these blind directions, and that is what is important. We do not need to know the directivity accurately in these blind directions.

The results for the three different evaluations of the aperture efficiency are shown in Fig. 6. It is worth mentioning that the

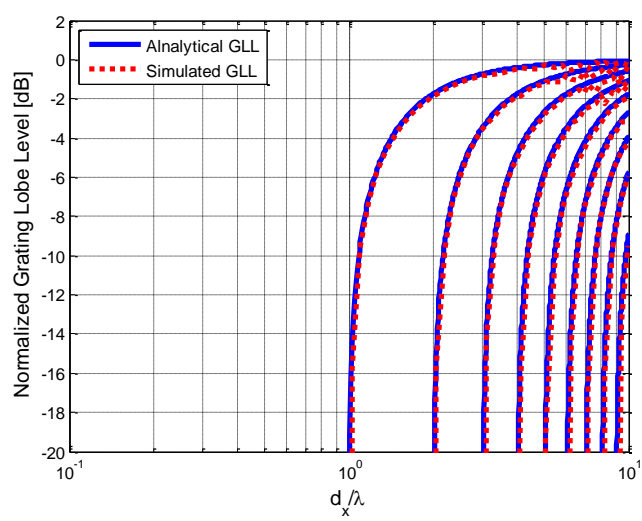

Fig. 9. Grating lobe level of $32 \times 32$-element slot array with different element spacing in H-plane. 


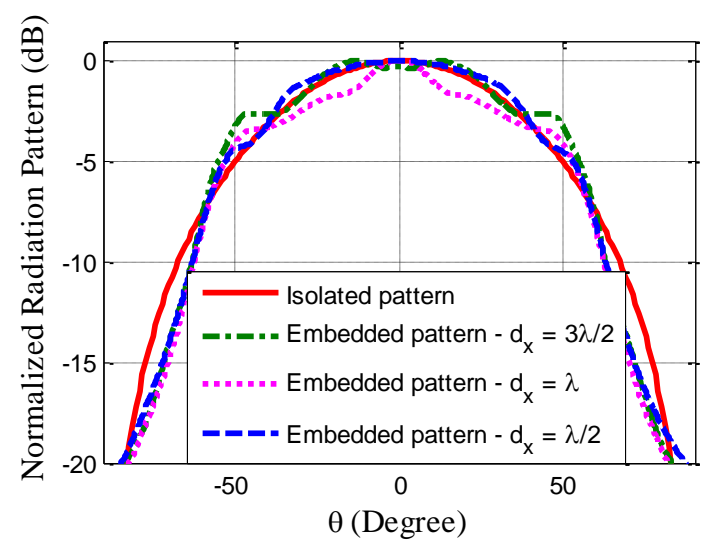

Fig. 10. Comparison of isolated and embedded element pattern with different element spacing in H-plane.

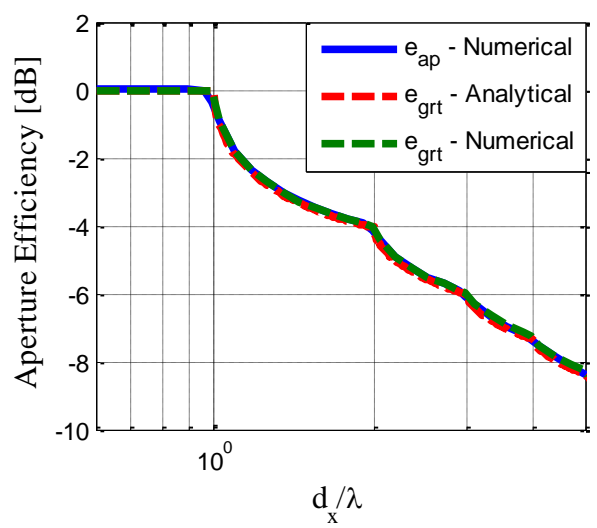

Fig. 11. Aperture efficiency of $32 \times 32$-element slot array with different element spacing in H-plane.

grating efficiency results are not reliable when the element spacing is an integer number of wavelengths, because there exist grating lobes along the ground plane with only one half of the lobes being in visible space. Thus, there is a transition region around these integer values of element spacing, corresponding to grating lobes changing from being evanescent to propagating. The extent of this transition region is $\pm \lambda /\left(32 d_{y}\right)$ for our $32 \times 32$ element array.

\section{B. Grating Lobes in H-plane}

Now we assume that grating lobes only appear in H-plane. The element spacing in $\mathrm{H}$-plane varies from $0.6 \lambda$ to $10 \lambda$, and the element spacing is fixed to $d y=0.67 \lambda$ in E-plane, contrary to our assumptions in the previous section. The spectrum of grating lobes for this case is shown in Fig. 7.

Fig. 8 illustrates the simulated directivity of the $32 \times 32$ slot array versus element spacing in $\mathrm{H}$ plane. The isolated slot farfield function in H-plane becomes by inserting (6) and (7) into (5), for $\varphi=0^{\circ}$ :

$$
G_{s l t}=C \times \cos \theta \times \cos \left(\frac{\pi}{2} \sin \theta\right) /\left[1-\sin \theta^{2}\right]
$$

where $\mathrm{C}$ is a constant. This pattern is zero along the ground plane (theta $=+/-90^{\circ}$ ) so that the grating lobes are suppressed

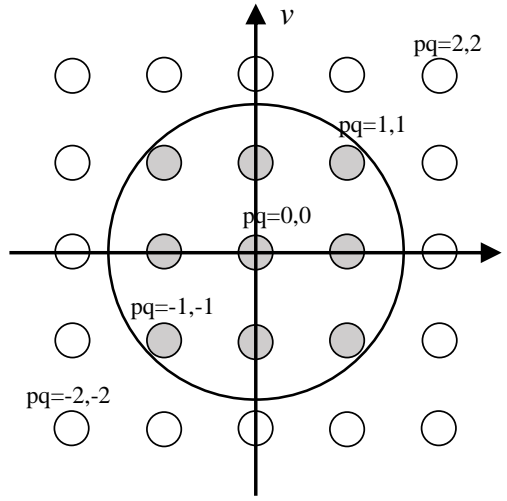

Fig. 12. Grating lobes and main beam spectrum.

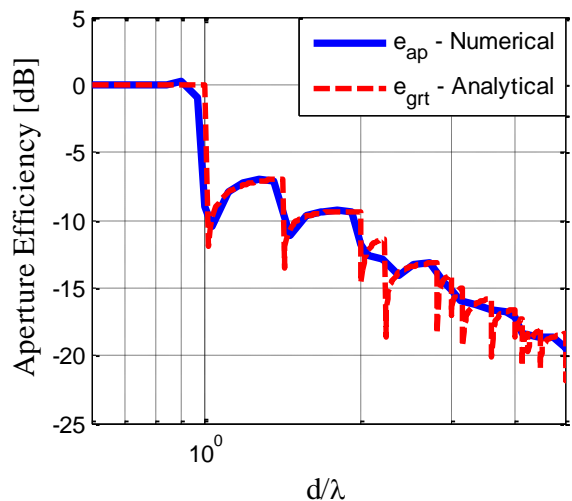

Fig. 13. Aperture efficiency of $32 \times 32$-element slot array for different spacing with equal spacing in $\mathrm{E}$ and $\mathrm{H}$-planes.

when they appear in visible space. Therefore, the transition to visible space is very smooth, so that sudden directivity reduction due to grating lobes is avoided.

Fig. 9 shows the simulated and analytical normalized grating lobe levels. The analytical grating lobe level is obtained by using (8) in (3). The analytical and simulated grating lobe levels have very good agreement in spite of the fact that the analytical grating lobe levels are calculated by using the isolated element pattern instead of the embedded element pattern. The H-plane isolated and embedded element patterns are shown in Fig. 10. The isolated and embedded patterns are more similar to each other than the patterns in Eplane because of the smaller mutual coupling in H-plane. Figs. 4 and 8 show that it is a quite good approximation to use the isolated element pattern instead of the embedded element pattern when calculating the grating lobe levels.

Fig. 11 shows the corresponding aperture efficiencies. Now we use (8) as the far-field function of the unit cell. The aperture efficiency does not drop dramatically when the element spacing is a fraction of the wavelength $\left(d_{x} / \lambda=1,2,3, \ldots\right)$, because the grating lobe levels appearing at grazing angle vanish due to the element pattern. The simulated and analytical results are in very good agreement. 


\section{Grating Lobes in All Planes}

Fig. 12 illustrates the general spectrum of grating lobes for an array with rectangular grid and equal element spacing. In this case we consider equal element spacings in $\mathrm{E}$ - and $\mathrm{H}$ planes. The numerical aperture efficiency obtained from the simulated directivity is compared with the analytical grating efficiency in Fig. 13. We see that the grating efficiency formula works very well even for this complex case. The grating efficiency can predict the aperture efficiency reduction due to the grating lobe.

\section{Grating Efficiency for Beam-Steered Array}

Beam-scanning is widely used with array antennas. This can cause undesirable grating lobes even when the element spacing is smaller than one wavelength. The directivity of array antenna decreases with $\cos \theta_{0}$ when the main beam is

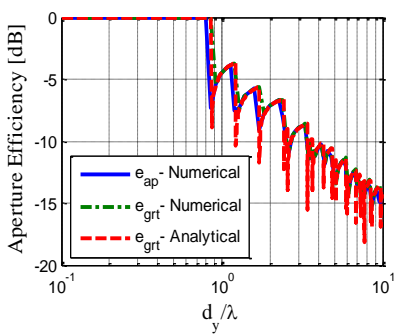

(a)

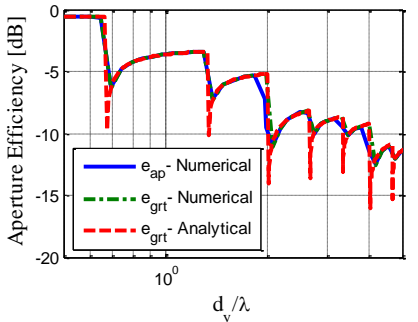

(c)

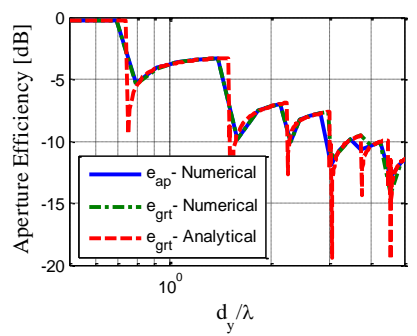

(b)

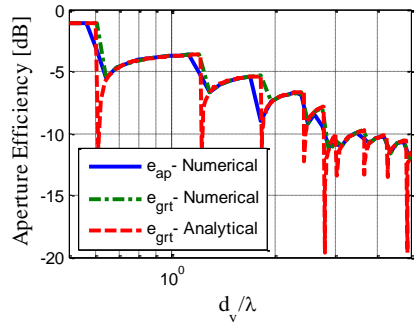

(d)
Fig. 14. Aperture efficiency of $32 \times 32$-element slot array for different steeríng angle in E-plane. (a) $10^{\circ}$. (b) $20^{\circ}$. (c) $30^{\circ}$. (d) $40^{\circ}$.

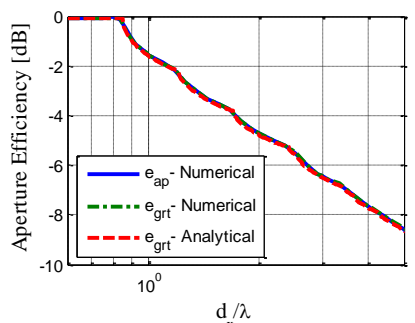

(a)

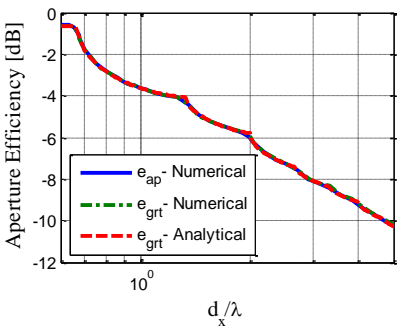

(c)

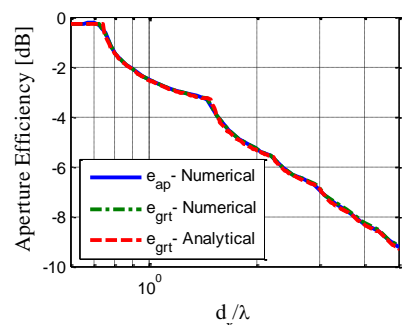

(b)

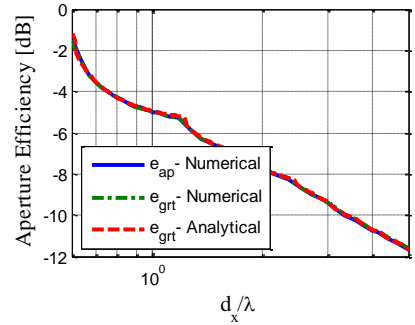

(d)
Fig. 15. Aperture efficiency of $32 \times 32$-element slot array for different steeríng angle in H-plane. (a) $10^{\circ}$. (b) $20^{\circ}$. (c) $30^{\circ}$. (d) $40^{\circ}$ steered an angle $\theta_{0}$ from broadside. This is due to the projection of the aperture into the observation direction [14]. Therefore, the grating efficiency in (3) is multiplied by the $\cos \theta_{0}$ projection factor when comparing with the numerical simulations.

Fig. 14 shows the aperture efficiency obtained from the simulated directivity and the grating efficiencies calculated both by numerical and analytical approaches, when the main beam is steered in E-plane. In this figure, the element spacing in E-plane varies from $0.1 \lambda$ to $10 \lambda$ While the element spacing is fixed to $d_{x}=0.67 \lambda$ in H-plane, similar to section $A$. The corresponding aperture efficiencies for steering the beam in $\mathrm{H}$ plane with a similar configuration as section $B$, is also shown in Fig. 15. Thus, the grating efficiency can predict the aperture efficiency reduction due to the grating lobe also in a phasesteered array.

\section{CONCLUSION}

We have presented and verified a simple formula called "grating efficiency" for calculating the aperture efficiency of a planar phased array antenna. The grating efficiency is the reduction in the aperture efficiency due to the power lost in the grating lobes. We used a uniformly excited array with slot elements to validate the accuracy of this formula. Although we verified the grating efficiency formula for a uniformly exited array of slots, it is also valid for tapered arrays. We have compared the analytical grating efficiency with aperture efficiency results obtained by simulations. We have shown that the grating efficiency formula is accurate even when we use the isolated element pattern to obtain the grating lobe levels, except near the blind angles when the grating lobes radiate along the ground plane in E-plane. We expect that the formula will be quite accurate also for other types of array elements that radiate from or above metal ground planes.

\section{REFERENCES}

[1] J. Yu, V. A. Khlebnikov, and M. Ka, "Wideband Grating-Lobe Suppression by Rotation of the Phased Array Stations in the SKA LowFrequency Sparse Aperture Array," accepted to IEEE Transactions on Antennas and Propagation, 2015.

[2] R. Y. Li, W. Jian, and L. T. Guang, "Research on Grating Lobe Reduction in Limited Scan Phased Array Antenna," Cross Strait QuadRegional Radio Science and Wireless Technology Conference (CSQRWC), Chengdu, July 2013.

[3] K. C. Kerby and J. T. Bernhard, "Sidelobe Level and Wideband Behavior of Arrays of Random Subarrays," IEEE Transactions on Antennas and Propagation, vol. 54, no.8, Aug. 2006.

[4] M. G. Bray, D. H. Werner, D. H. Boeringer, and D. W. Machuga, "Optimization of thinned aperiodic linear phased arrays using genetic algorithms to reduce grating lobes during scanning, "IEEE Transactions on Antennas and Propagation, vol. 50, no. 12, pp. 1732-1742, Dec. 2002.

[5] Z. Ding, W. Gao, J. Liu, T. Zeng, and T. Long, "A Novel Range Grating Lobe Suppression Method Based on the Stepped-Frequency SAR Image," IEEE Geoscience and Remote Sensing Letters, vol. 12, no. 3, March 2015.

[6] N. Levanon and E. Mozeson, "Nullifying ACF grating lobes in stepped frequency train of LFM pulses," IEEE Transactions on Aerospace and Electronic Systems, vol. 39, no. 2, pp. 694-703, Apr. 2003. 
[7] P.-S. Kildal, A. Uz Zaman, E. Rajo-Iglesias, E. Alfonso, A. ValeroNogueira, "Design and experimental verification of ridge gap waveguides in bed of nails for parallel plate mode suppression," IET Microwaves, Antennas \& Propagation, vol. 5, no. 3, pp. 262-270, March 2011.

[8] H. Raza, J. Yang, P.-S. Kildal, E. Alfonso, "Microstrip-ridge gap waveguide - study of losses, bends and transition to WR-15," IEEE Transactions on Microwave Theory and Techniques, vol. 62, no. 9, pp. 1943 - 1952, Sept. 2014.

[9] E. Pucci, E. Rajo-Iglesias, J.-L. Vazquez-Roy and P.-S. Kildal, "Planar dual-mode horn array with corporate-feed network in inverted microstrip gap waveguide," IEEE Transactions on Antennas and Propagation, vol. 62, no. 7, pp- 3534 - 3542, July 2014.

[10] Y. Miura, J. Hirokawa, M. Ando, Y. Shibuya and G. Yoshida, "Doublelayer full -corporate-feed hollow-waveguide slot array antenna in the 60 GHz band," IEEE Trans. Antennas Propag., vol. 59, no. 8, pp. 28442851, Aug. 2011.

[11] S. A. Razavi, P.-S. Kildal, L. Xiang and E. Alfonso, "2×2-slot element for $60 \mathrm{GHz}$ planar array antenna realized on two doubled-sided PCBs using SIW cavity and EBG-type soft surface fed by microstrip-ridge gap waveguide," IEEE Transactions on Antennas and Propagation, vol. 62, no. 9, pp. 4564 - 4573, Sept. 2014.

[12] T. J. Brockett and Y. Rahmat-Samii, "Subarray design diagnostics for the suppression of undesirable grating lobes," IEEE Trans. Antennas Propag., vol. 60, no. 3, pp. 1373-1380, March 2012.

[13] D. Blanco, N. Llombart, E. Rajo-Iglesias, "On the use of leaky wave phased arrays for the reduction of the grating lobe level," IEEE Trans. Antennas Propag., vol. 62, no. 4, pp. 1789-1795, April 2014.

[14] P. S. Kildal, Foundations of Antennas: A Unified Approach for Line-ofSight And Multipath, Kildal Antenn AB, 2015 (free PDF available at www.kildal.se).

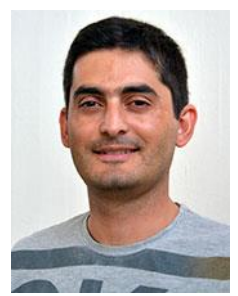

Abbas Vosoogh received the B.Sc. degree in electrical engineering from the University of Sistan and Baluchestan, Zahedan, and the M.Sc. degree from K. N. Toosi University of Technology, Tehran, Iran. He is currently pursuing his Ph.D. degree with Chalmers University of Technology, Gothenburg, Sweden. His current research interests include the development of gap waveguide technology for millimeter and submillimeter wave applications, microwave passive components, EBG, soft and hard surfaces and $60 \mathrm{GHz}$ planar array antennas based on Gap technology.

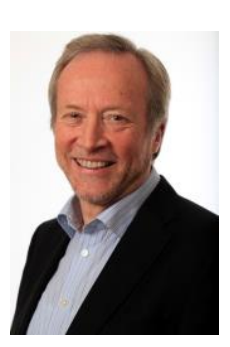

Per-Simon Kildal (M'82-SM'84-F'95) has been Professor at Chalmers University of Technology, Gothenburg, Sweden since 1989. He is now heading the Division of Antenna Systems at Department of Signals and Systems at Chalmers.

Prof Kildal received two best paper awards for articles published in the IEEE Transactions on Antennas and Propagation, and he was the recipient of the 2011 Distinguished Achievements Award of the IEEE Antennas and Propagation Society.

Kildal has authored an antenna textbook, and more than 150 journal articles and letters, most of them in IEEE or IET journals. He has designed two very large antennas, including the Gregorian dual-reflector feed of the Arecibo radiotelescope. He has invented several reflector antenna feeds, the latest being the so-called "Eleven antenna".

Kildal is the originator of the concept of soft and hard surfaces, recently resulting in the gap waveguide, a new low-loss metamaterial-based transmission line advantageous in particular above $30 \mathrm{GHz}$. Kildal has received large individual grants from the Swedish research council VR and from the European Research Council ERC for research on gap waveguides.

His research group has pioneered the reverberation chamber into an accurate measurement tool for antennas and wireless terminals subject to Rayleigh fading. This has been successfully commercialized in Bluetest AB. 